\title{
ANDES

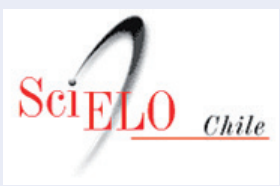

www.scielo.cl

\section{Revista Chilena de Pediatría en el mundo global: Andes Pediatrica 2021}

\author{
Revista Chilena de Pediatría in the global world: \\ Andes Pediatrica 2021
}

\author{
Francisco Cano Sch. ${ }^{a}$, Luisa Schonhaut B. ${ }^{a}$, Paul R. Harris D. ${ }^{a}$
}

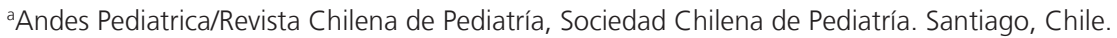

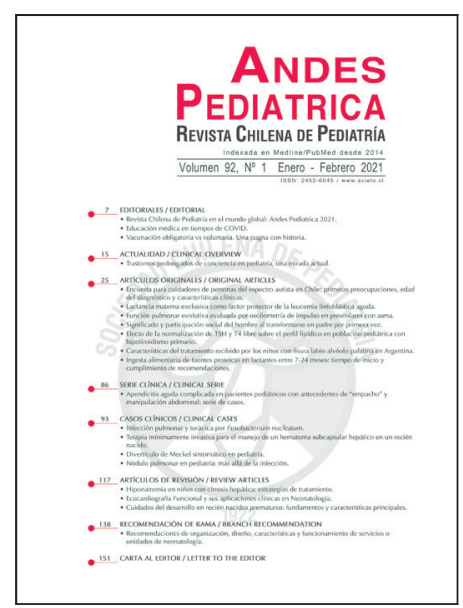

A propósito de la pandemia COVID-19 hemos vivido un año de profundos cambios. Dado que más que espectadores, somos protagonistas, nos es difícil evaluar estos cambios, dimensionarlos y proyectarlos. Mientras estamos dentro de la tormenta que destruye todo a su paso, es difícil sacar conclusiones de lo que está pasando. Pero todos sabemos que el mundo ha cambiado. Sabemos que han cambiado nuestras tareas y acciones del día a día, que ha cambiado nuestra forma de comunicarnos, que ha cambiado hasta el sentido del tiempo en lo cotidiano. Pero lo más importante, han cambiado nuestras prioridades, ha cambiado la importancia que dábamos a muchas cosas, y hoy sabemos que lo que dijo John Lennon hace muchos años, era cierto. Cuando le preguntaron qué era para él la Vida, dijo "...la Vida es lo que pasa por el lado de nosotros, mientras estamos ocupados de cosas muy importantes".

Quizás una reflexión que ha tardado un tiempo en ser asumida, y que probablemente no es aún reconocida por todos, es lo que dijo Yuval Harari al analizar esta epidemia. Escribió (no traduzco para no quitar el sentido)..." In this time of crisis, we are faced with two particularly important decisions. The first is between totalitarian surveillance and citizen empowerment. The second is between nationalist isolation and global solidarity."
El año 1998 Revista Chilena de Pediatría (RCHP) ingresó a la colección SciELO Chile, y el 2014 se incorporó a la National Library of Medicine (NLM) con su extendido motor de búsqueda PubMed. Durante los últimos años, importantes cambios orientados a optimizar el proceso editorial, la calidad de las publicaciones y ampliar el área de influencia incluyeron cambios en el Comité Editorial, que consideró la incorporación de un asesor Bioinformático experto en publicación científica, un Comité Editorial Internacional, 3 Editoras junior, la traducción al inglés de los artículos originales, la publicación de manuscritos venideros, y la adquisición de un identificador de objeto digital propio (DOI).

En el tránsito a un futuro digital e interconectado, el Proyecto "Revista Chilena de Pediatría 2020: globalización e impacto científico" fue concursado y obtenido en el concurso Fondo de Publicación de Revistas Científicas Chilenas, CONICYT 2019. Este proyecto considera dentro de otros objetivos, homologar y actualizar los metadatos de los manuscritos para que los artículos de la revista compartan temáticas, y sean capaces de vincularse con otros artículos relacionados, además del acceso a servicios de la web de uso internacional para el reconocimiento de los revisores y para la detección de plagio.

La presencia de la revista, su difusión y acceso a los

Correspondencia:

Francisco Cano Sch.

fcanosch@gmail.com 
artículos en las Redes Sociales ha sido considerado de alta relevancia, como una forma de alcanzar un público de lectores que ha abandonado los tradicionales canales de acceso a la información, y como profesionales de la salud adquieren la información en forma virtual desde plataformas interactivas que no reconocen fronteras.

A eso se refiere Harari al decir que una decisión clave es optar por un mundo global, y no un aislamiento nacionalista. Ese concepto viene desde hace mucho, mucho tiempo. Ese concepto fue clave en la evolución humana. La interacción entre grupos humanos cada vez mayores, que se contó al principio en cientos, luego miles y millones, hoy nos muestra una realidad global que en este tiempo de pandemia ha dado un salto exponencial, ahorrando decenas de años a este proceso. Esto, indica Harari, es posible solamente si un "gran número de extraños cree en una idea común". Cuando decenas de científicos participan en un proyecto desde diferentes lugares del mundo, lo hacen tras una idea común. Cuando miles de profesionales de diferentes lugares del mundo participan de una publicación científica, lo hacen porque consideran que esa publicación responde a intereses y objetivos comunes. Esa es la base de la globalidad, y funciona desde el principio de los tiempos ${ }^{1}$. En el caso de una publicación pediátrica nacional, de un país que hasta hace pocas décadas se mantenía lejos de la globalización, RCHP con 90 años de vida, ha sido parte de este tiempo de cambio (figura 1$)^{2}$.
Los esfuerzos y logros detallados previamente nos sorprendieron al entender que la visibilidad de este órgano traspasaba nuestras fronteras. Prueba de ello es el registro de accesos a la Revista informado por la plataforma Crossref (Crossref.org), plataforma editorial de la cual RCHP forma parte desde hace 2 años, que muestra que de 8.400 accesos via DOI a fines del 2019, contabilizamos mas de 23.000 accesos a fines del 2020. Al contabilizar los accesos vía plataforma OJS, de 42.000 accesos el año 2017, se registran mas de 130.000 el año 2020, provenientes fundamentalmente de Chile, Estados Unidos, Perú, Colombia, México, Argentina y España. Prueba de ello es también el creciente número artículos publicados y en particular, de manuscritos provenientes del extranjero, de toda Latinoamérica, y de otros continentes no-hispanoparlantes. Entre los manuscritos publicados el año 2020, en un tercio participaron autores con filiaciones extranjeras y un cuarto de los autores pertenecen a disciplinas diferentes a la medicina, como son kinesiología, psicología, enfermería, entre otros.

La colaboración científica entre ditintas instituciones, países y profesiones va consolidando la producción científica, aumenta el impacto y visibilidad, otorgando una mirada transdisciplinar e internacional de la investigación pediátrica. El índice de colaboración internacional para el 2019 fue 17\%, lo que muestra una importante tendencia al ascenso respecto a años previos. En las figuras 2 y 3 se muestran las redes de colaboración de instituciones nacionales y las interna-

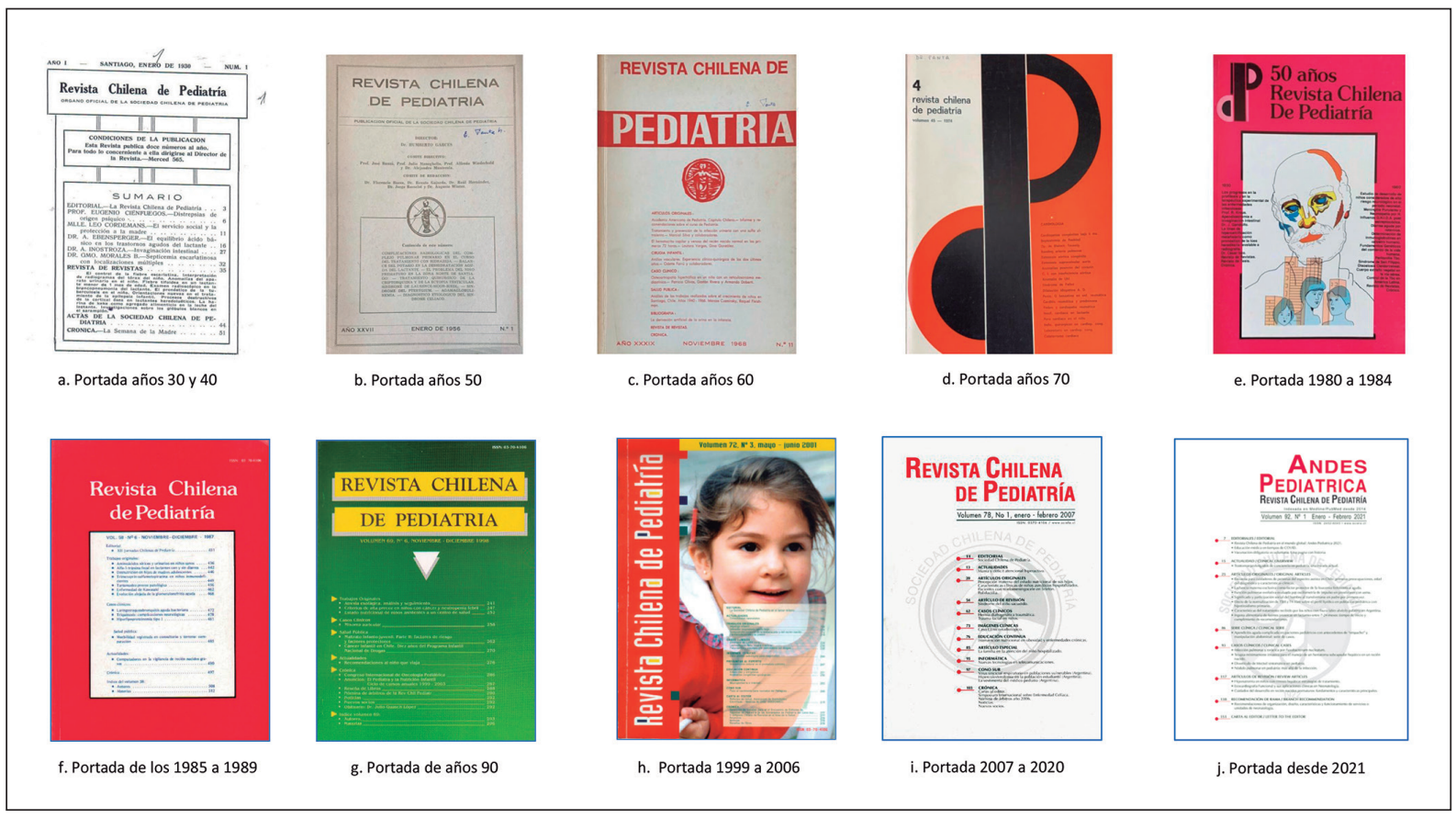

Figura 1. Portadas Revista Chilena de Pediatría desde 1930 a la fecha 

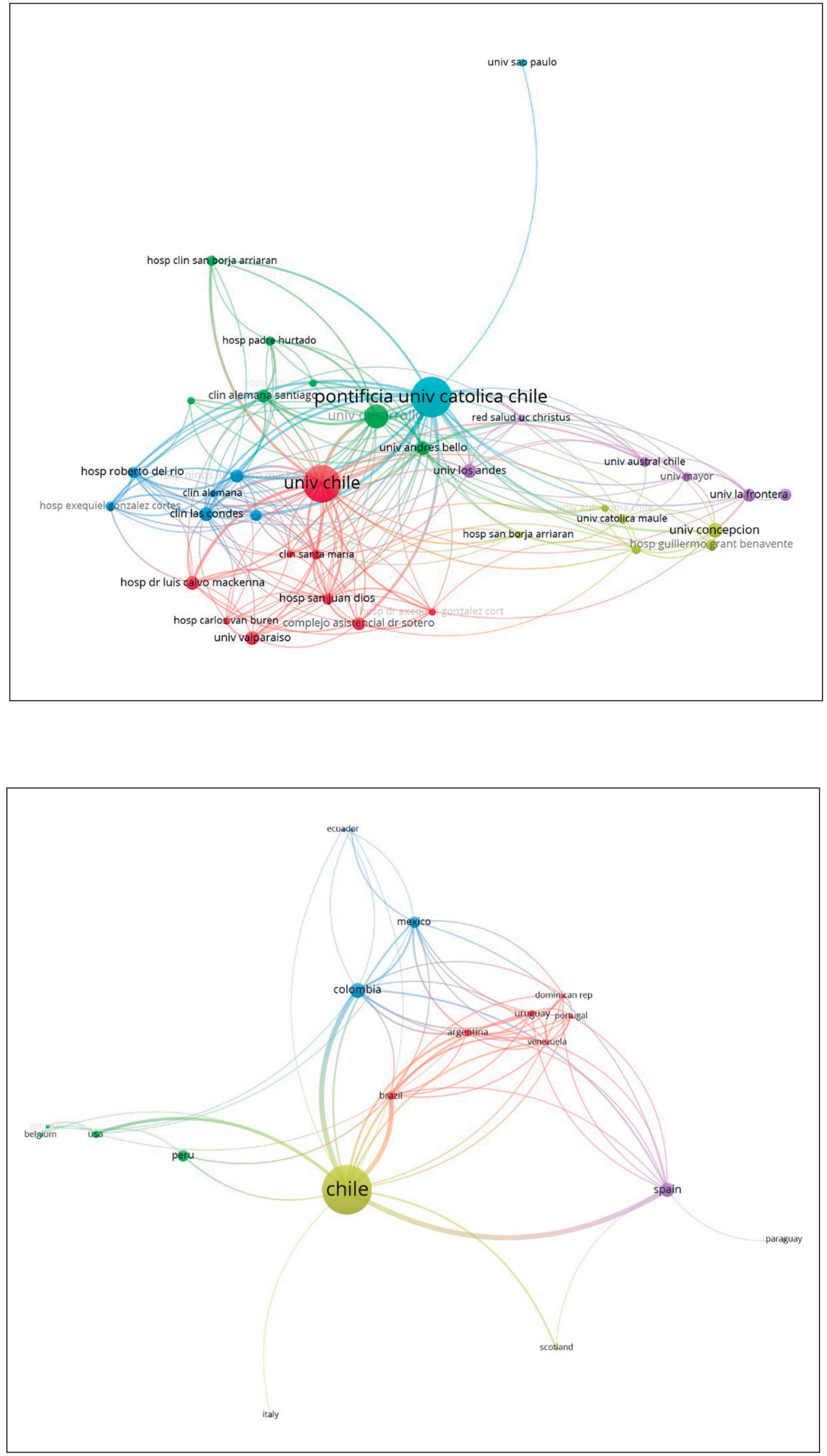

Figura 2. Cluster de instituciones que publican en Revista Chilena de Pediatría y sus co-autorías, entre los años 2015-2020. El tamaño de los nodos corresponde a aquellas instituciones que tiene mayor cantidad de nexos colaborativos, el grosor de las líneas representa la intensidad y fortaleza de las relaciones establecidas. Fuente Web of Science. Nota: Una institución puede aparecer dividida dependiendo de cómo los autores inscriben la filiación, lo que resta representatividad a la institución
Figura 3. Cluster de países que publican en Revista Chilena de Pediatría y sus co-autorías, entre los años 2015-2020. El tamaño de los nodos corresponde a aquellas instituciones que tiene mayor cantidad de nexos colaborativos, el grosor de las líneas representa la intensidad y fortaleza de las relaciones establecidas. Fuente Web of Science. 
cionales respectivamente, lo que sin duda demuestra que RCHP ha atravesado las fronteras de este lejano país andino. En la actualidad se publican 303 revistas pediátricas de corriente principal, siendo 12 de ellas latinoamericanas, RCHP ocupa el 60 lugar entre las Revistas Pediátricas Latinoamericanas según el Ranking Scimago $2019^{3}$.

El mundo ha cambiado a una velocidad insospechada en las últimas décadas, y este proceso ha sido acelerado por la pandemia COVID-19. La globalización de la investigación y de la publicación científica son particularmente importantes y parte de ello, y al revisar los títulos de las principales revistas pediátricas del mundo, nos damos cuenta de que muchas instituciones ya lo habían considerado, asumiendo una denominación que traspasaba las fronteras, aunque sin perder su identidad. Revistas como Pediatrics, Journal of Pediatrics, Neonatology, Child Development, Infants, Maternal and Child Nutrition, Archives of Disease in Childhood, y muchas otras, se han asumido como parte de un mundo global que convoca a miles de lectores que forman una comunidad interconectada que se mantiene unida y cooperando porque la idea común que comparten los ha hecho crecer y dejar atrás los pequeños grupos de intereses de décadas pasadas. El mundo actual solo tiene fronteras ficticias, creadas por intereses locales, que son traspasadas por las ideas y proyectos multicéntricos, y en una época de catástrofe global esto ha sido probablemente el mayor cambio, quizás el único aspecto positivo que este tiempo de enfermedad y muerte ha aportado. Al igual que aquellos Journals que se presentan como parte de este mundo global, Andes Pediatrica/Revista Chilena de Pediatría, desde el 2021, será la forma en que nos verán en el mundo y en las redes que hoy lo conforman.

\section{Conflicto de intereses}

Los autores declaran no tener conflicto de intereses.

\section{Agradecimientos}

En la presente editorial queremos dar un especial agradecimientos la Sr. Italo Costa-Roldan, Licenciado en Biología de la Pontificia Universidad Católica de Valparaíso, por su permanente asesoría bioinformática y por la elaboración de las figuras del presente manuscrito.

\section{Referencias}

1. Yuval Noa Harari, De animales a dioses. El Arbol del Saber, $1^{\text {a }}$ Ed, Buenos Aires, Debate,2014, ISBN978-987-
3572-13-1.

2. Schonhaut L, Millan T, Zepeda A. Revista Chilena de Pediatría: 90 años de historia. Rev Chil Pediatr. 2020;91(1): 139-145.
3. Ranking Scimago 2019 [consultado 30.12.20] disponible en https:// www.scimagojr.com/journalrank. php?area $=2700 \&$ country $=$ Latin $\% 20$ America\&category $=2735$. 